This is the final peer-reviewed accepted manuscript of:

Gobbi, F., Kolev, N., \& Mulinacci, S. (2019). JOINT LIFE INSURANCE PRICING USING EXTENDED MARSHALL-OLKIN MODELS. ASTIN Bulletin, 49(2), 409-432. doi:10.1017/asb.2019.3.

The final published version is available online at: https://doi.org/10.1017/asb.2019.3

Rights / License:

The terms and conditions for the reuse of this version of the manuscript are specified in the publishing policy. For all terms of use and more information see the publisher's website. 


\title{
Joint Life Insurance Pricing Using Extended Marshall-Olkin Models
}

\author{
Fabio Gobbi*, Nikolai Kolev ${ }^{\dagger}$ Sabrina Mulinacci ${ }^{\ddagger}$
}

December 27, 2018

\begin{abstract}
In this paper we suggest a modeling of joint life insurance pricing via Extended Marshall-Olkin models and related copulas. These models are based on the combination of two approaches: the absolutely continuous copula approach, where the copula is used to capture dependencies due to environmental factors shared by the two lives, and the classical Marshall-Olkin model, where the association is given by accounting for a fatal event causing the simultaneous death of the two lives. New properties of the Extended Marshall-Olkin model are established and applied to a sample of censored residual lifetimes of couples of insureds extracted from a dataset of annuities contracts of a large Canadian life insurance company. Finally, some joint life insurance product is analyzed.
\end{abstract}

Keywords: Bivariate Marshall-Olkin's model, Copula, Mortality intensity, Singularity, Joint life insurance products.

\section{Introduction}

Insurance policies involving multiple lives are natural influenced by the dependence structure among the insured lifetimes. This is particularly true for married couples involved in joint life and last survivor insurance contracts. While classical actuarial practice advocates independence among involved remaining lifetimes (because of computational convenience), it is well recognized and confirmed by evidence that the independence belief is naive, unrealistic and leads to a wrong estimate of the associated insurance premiums. As a consequence, in recent past a wide stream of actuarial literature has been focused on the problem of modeling the dependence between remaining lifetimes of married couples and on the analysis of the impact of such dependence on the premium calculation of insurance policies.

${ }^{*}$ Department of Statistics, University of Bologna, Italy

${ }^{\dagger}$ Department of Statistics, University of São Paulo, Brazil

${ }^{\ddagger}$ Department of Statistics, University of Bologna, Italy 
Possible sources of dependence are common lifestyle, bereavement (broken-heart) effects and catastrophic exogenous events causing the simultaneous death of both the spouses. In existing literature, essentially two approaches have been utilized: the semi-Markov chain approach and the copula approach. The semi-Markov approach is used in Denuit et al. (2001), Spreeuw and Wang (2008), Spreeuw and Owadally (2013) and in Ji et al. (2011) where four states are considered: both spouses are alive, husband is dead and wife is alive, wife is dead and husband is alive and both spouses are dead. In Ji et al. (2011) also the transition from the state in which both spouses are alive to that in which both are dead is allowed and since the force of mortality of a widower is assumed to be dependent on the time passed from the death of the other spouse, the model accounts for external events causing the simultaneous death and for the broken-heart effect. The copula approach was firstly adopted in Frees et al. (1996) where a dataset on annuities contracts on the joint life of couples provided by a Canadian insurer is analyzed. Many papers have been then devoted to the use of copula functions to model the dependence among the lifetimes in a couple and most of them focus on the absolutely continuous copulas (mainly Archimedean copulas): see Youn and Shemyakin (1999), and Shemyakin and Youn (2006), Carriere (2000), Luciano et al. (2008), Luciano et al. (2016), Spreeuw (2006), Denuit et al. (2001), Dufresne et al. (2018). As for the broken-heart syndrome, in addition to the multistate Markov chain method already mentioned, a frailty based approach has been considered in Gouriéroux and Lu (2015): this tool has been further generalized and estimated on a French reinsurer database in Lu (2017).

In this paper we will apply the copula based approach to model the joint life insurance pricing. Unfortunately, assuming an absolutely continuous copula, the resulting model is not able to capture the occurrence of simultaneous deaths caused by fatal events. Taking into account these killing events, the common shock model has been applied in Frees et al. (1996) where it is compared to the absolutely continuous copula approach. Denuit et al. (2006) investigated the dependence among coupled lifetimes through a Generalized Marshall-Olkin model (deeply studied by Li and Pellerey, 2011) that extends the classical Marshall-Olkin's one (see Marshall and Olkin, 1967).

To proceed, let $T^{m}$ and $T^{f}$ be non-negative continuous random variables representing the remaining lifetimes of a husband and his wife. The Marshall-Olkin's probabilistic construction consists in modeling $T^{m}$ and $T^{f}$ through a stochastic representation

$$
\left(T^{m}, T^{f}\right)=\left(\min \left(X^{m}, Z\right), \min \left(X^{f}, Z\right)\right),
$$

where $X^{m}, X^{f}$ and $Z$ are some independent non-negative random variables, $Z$ being the time of occurrence of the event killing both individuals. In general, the relation (1) implies that the distribution of $\left(T^{m}, T^{f}\right)$ has a singularity along the main diagonal in the first quadrant, due to the fact that $P\left(T^{m}=T^{f}\right)>0$. In order to describe the dependencies in the joint life survival status, the model (1) has been applied 
by many authors (see Chapter 9 of Bowers et al. (1997), Denuit et al. (2006) and references therein).

The stochastic representation (1) has been recently improved by Kolev and Pinto (2018) considering the relation

$$
\left(T^{m}, T^{f}\right)=\left(\min \left(X^{m}, Z\right), \min \left(X^{f}, Z / \beta\right)\right),
$$

where $\beta \in(0,1]$. One can observe that the common shock identified by the random variable $Z$ has an immediate killing effect on husband's life while the corresponding impact on the life of the spouse is delayed. A similar scenario has been investigated in Lee and Cha (2018) where a dynamic common shock model is introduced and studied. An external shock process is assumed so that at the arrival time of each shock not necessarily both individuals die, but the effect of the occurrence of the shock for the survived individual results in a change in his/her mortality rate.

Along with other properties of the Generalized Marshall-Olkin model (1) applied to joint life insurance, Denuit et al. (2006) study the impact of existing dependence between remaining lifetimes of married couple on the net single premium for a continuous $n$-year joint life annuity (paying $1 \$$ per year as long as both spouses survive) given by

$$
\bar{a}_{x y ; \bar{n} \mid}=\int_{t=0}^{n} \exp \left(-\int_{0}^{t} \delta_{u} d u\right) \bar{F}_{T^{m}, T^{f}}(t, t) d t,
$$

where $\delta_{u}$ is the instantaneous interest rate at time $u$ and $\bar{F}_{T^{m}, T^{f}}$ is the joint survival function of random variables $T^{m}$ and $T^{f}$.

Because of the common lifestyle (husband and wife share the same common environment) it is reasonable to assume that "individual shocks" identified by $X^{m}$ and $X^{f}$ in (1) are dependent random variables. More specifically, let the distribution of the pair $\left(X^{m}, X^{f}\right)$ be defined via the joint survival function $\bar{F}_{X^{m}, X^{f}}(x, y)=P\left(X^{m}>\right.$ $x, X^{f}>y$ ) for $x, y \geq 0$, and consider a continuous non-negative random variable $Z$ with survival function $\bar{F}_{Z}(x)=P(Z>x)$, independent of $X^{m}$ and $X^{f}$. Thus, we arrive to the Extended Marshall-Olkin model introduced in Pinto and Kolev (2015) and defined as follows

$$
\bar{F}_{T^{m}, T^{f}}(x, y)=P\left(T^{m}>x, T^{f}>y\right)=\bar{F}_{X^{m}, X^{f}}(x, y) \bar{F}_{Z}(\max \{x, y\}), \quad x, y \geq 0 .
$$

Our aim is to apply Extended Marshall-Olkin distributions generated by relation (3) in modeling joint life insurance pricing. The associated Extended Marshall-Olkin copula will be obtained, joining the marginals which could be Gompertz, Makeham or Weibull distributed, being most appropriate for fitting real data for population of age over 60 (see Bowers et al., 1997).

Let us note that in the conclusions of Denuit et al. (2006), the authors suggest another version of the model based on a frailty approach to describe common lifestyle 
factors, implying an underlying dependence of Archimedean type which combines with the fatal shock induced by the random variable $Z$ in the stochastic representation (1). This idea is incorporated in the Extended Marshall-Olkin model. A frailty approach was further developed in Gouriéroux and $\mathrm{Lu}$ (2015).

The plan of the paper is the following. In section 2 we discuss new properties of the Extended Marshall-Olkin model (3) and associated copula. In section 3 we show the results of an empirical application and in section 4 we analyze some joint life insurance products under model (3). Section 5 concludes.

\section{$2 \quad$ Extended Marshall-Olkin Model}

In this section we present the Extended Marshall-Olkin model introduced and studied in Pinto and Kolev (2015). It will be applied to model the residual lifetimes of a couple involved in a joint life insurance contract. The relevance of the model is justified by the fact that the induced dependence structure incorporates the common lifestyle and the possibility of the occurrence of the simultaneous death of the two individuals in the couple.

Let $(\Omega, \mathcal{F}, \mathbb{P})$ be a probability space and let $T_{x_{m}}^{m}=T^{m}$ and $T_{x_{f}}^{f}=T^{f}$ be two continuous random variables denoting the residual lifetimes of a male and a female in a couple that enter in a joint life insurance contract at ages $x_{m}$ and $x_{f}$, respectively. In order to model the dependence structure of the vector $\left(T^{m}, T^{f}\right)$ from the entry ages $x_{m}$ and $x_{f}$, respectively, we consider three non-negative continuous underlying random variables $X^{m}, X^{f}$ and $Z$ connected by the stochastic representation (1). We assume that the two lifetimes $X^{m}$ and $X^{f}$ are dependent, because the two individuals in the couple share the same lifestyle, the same economic status and so on. In addition, some independent rare fatal event can cause their simultaneous death at time $Z$. Therefore, the joint survival function $\bar{F}_{T^{m}, T^{f}}$ of the vector $\left(T^{m}, T^{f}\right)$ is specified by (3), i.e., by the Extended Marshall-Olkin (EMO) model launched by Pinto and Kolev (2015).

In what follows, we will discuss the flexibility of the EMO model (3) and its advantages with respect to the Generalized Marshall-Olkin model (where $X^{m}$ and $X^{f}$ are assumed to be independent). Several new properties of the EMO model and associated copula related to joint life insurance will be established, including the analysis of mortality intensities.

\subsection{Comparisons with the Generalized Marshall-Olkin model}

If the underlying distribution of the vector $\left(X^{m}, X^{f}\right)$ is positive quadrant dependent, that is $\bar{F}_{X^{m}, X^{f}}(x, y) \geq \bar{F}_{X^{m}}(x) \bar{F}_{X^{f}}(y)$ for all $x, y \geq 0$, then the resulting EMO distribution generated by (3) dominates the Generalized Marshall-Olkin type 
distribution of Li and Pellerey (2011), which can be written as

$$
\bar{F}_{T^{m}, T^{f}}^{G M O}(x, y)=\bar{F}_{X^{m}}(x) \bar{F}_{X^{f}}(y) \bar{F}_{Z}(\max (x, y)) .
$$

Since the Generalized Marshall-Olkin distributions are positive quadrant dependent, then this property is satisfied by the EMO distributions as well.

This conclusion might fail under the EMO model if the vector $\left(X^{m}, X^{f}\right)$ is negative quadrant dependent as the following example shows.

Example 2.1. Let $\left(X^{m}, X^{f}\right)$ be distributed according to the Gumbel's type I bivariate exponential distribution

$$
\bar{F}_{X^{m}, X^{f}}(x, y)=\exp \{-x-y-\theta x y\}, \quad \theta \in[0,1], x, y \geq 0,
$$

which is negative quadrant dependent since

$$
\bar{F}_{X^{m}, X^{f}}(x, y)=\exp \{-x-y-\theta x y\} \leq \exp \{-x-y\}=F_{X^{m}}(x) F_{X^{f}}(y) .
$$

Let $Z$ be exponentially distributed with parameter $\lambda>0$. Then the resulting EMO distribution (3) is given by

$$
\bar{F}_{T^{m}, T^{f}}(x, y)=\exp \{-x-y-\theta x y-\lambda \max (x, y)\} .
$$

If $x \leq y$, it can be easily checked that

$$
\bar{F}_{T^{m}, T^{f}}(x, y) \geq \bar{F}_{T^{m}}(x) \bar{F}_{T^{m}}(y)=e^{-(1+\lambda)(x+y)}
$$

if and only if $y \in\left[0, \frac{\lambda}{\theta}\right]$. Since a similar result holds for $x>y$, we have that

$$
\bar{F}_{T^{m}, T^{f}}(x, y) \geq \bar{F}_{T^{m}}(x) \bar{F}_{T^{m}}(y)
$$

holds if and only if $0<x \leq \frac{\lambda}{\theta}$ and $0<y \leq \frac{\lambda}{\theta}$.

Therefore, although the stochastic representation (1) induces a positive dependence between random variables $T^{m}$ and $T^{f}$ (because of the common shock presence), the negative quadrant dependence of the vector $\left(X^{m}, X^{f}\right)$ might result in a vector $\left(T^{m}, T^{f}\right)$ which is neither positive quadrant dependent nor negative quadrant dependent.

An interesting relationship between the EMO model (3) and associated Sibuya's dependence function is given below.

Remark 2.1. Denote by $r_{X^{i}}$ the force of mortality (hazard rate) associated with the random variable $X^{i}$ and let $H_{X^{i}}$ be the corresponding cumulative hazard rate for $i=m, f$, i.e.,

$$
\bar{F}_{X^{i}}(x)=P\left(X^{i}>x\right)=\exp \left\{-H_{X^{i}}(x)\right\}=\exp \left\{-\int_{0}^{x} r_{X^{i}}(u) d u\right\}, \quad i=m, f .
$$


We obtain from (3) that $H_{T^{i}}(x)=H_{X^{i}}(x)+H_{Z}(x), i=m, f$, where

$$
\bar{F}_{Z}(x)=P(Z>x)=\exp \left\{-H_{Z}(x)\right\}=\exp \left\{-\int_{0}^{x} r_{Z}(u) d u\right\} .
$$

This exponential representation in the bivariate case can be written as follows

$$
\bar{F}_{X^{m}, X^{f}}(x, y)=\exp \left\{-H_{X^{m}}(x)-H_{X^{f}}(y)+D_{X^{m}, X^{f}}(x, y)\right\}, \quad x, y \geq 0,
$$

where $D_{X^{m}, X^{f}}(x, y)=\ln \frac{\bar{F}_{X^{m}, X^{f}}(x, y)}{\bar{F}_{X^{m}(x) \bar{F}_{X f}(y)}}$ is Sibuya's dependence function introduced by Sibuya (1960), satisfying the boundary conditions $D_{X^{m}, X^{f}}(x, 0)=D_{X^{m}, X^{f}}(0, y)=0$. Note that $D_{X^{m}, X^{f}}(x, y)$ is positive or negative, if and only if the vector $\left(X^{m}, X^{f}\right)$ is positive or negative quadrant dependent, respectively.

Thus, the EMO model can be equivalently rewritten as

$$
\bar{F}_{T^{m}, T^{f}}(x, y)=\exp \left\{-H_{X^{m}}(x)-H_{X^{f}}(y)+D_{X^{m}, X^{f}}(x, y)-H_{Z}(\max (x, y))\right\} .
$$

Denuit et al. (2006) establish in their section 2.3 that, if $X^{m}$ and $X^{f}$ are independent and $y>x$, then

$$
P\left(T^{f}>y \mid T^{m}>x, T^{f}>x\right)=P\left(T^{f}>y \mid T^{f}>x\right) .
$$

In other words, under the Generalized Marshall-Olkin, model the survival of $T^{m}$ to time $x$ is irrelevant for the survival of the female to time $y$ if $T^{f}>x$. We revise the last equality in the EMO model in the next statement.

Proposition 2.1. Let $\left(T^{m}, T^{f}\right)$ follow the EMO model (3) and let us suppose that $y>x$. The following inequalities are equivalent

$$
\begin{gathered}
P\left(T^{f}>y \mid T^{m}>x, T^{f}>x\right) \leq P\left(T^{f}>y \mid T^{f}>x\right) \\
P\left(X^{f}>y \mid X^{m}>x, X^{f}>x\right) \leq P\left(X^{f}>y \mid X^{f}>x\right) \\
P\left(X^{m}>x \mid X^{f}>y\right) \leq P\left(X^{m}>x \mid X^{f}>x\right) .
\end{gathered}
$$

Proof. Using relation (5) we obtain

$$
\begin{aligned}
P\left(T^{f}>y \mid T^{m}>x, T^{f}>x\right) & =\frac{\bar{F}_{X^{m}, X^{f}}(x, y) \bar{F}_{Z}(y)}{\bar{F}_{X^{m}, X^{f}}(x, x) \bar{F}_{Z}(x)}= \\
& =\frac{\bar{F}_{X^{m}, X^{f}}(x, y) \bar{F}_{Z}(y) \bar{F}_{X^{f}}(y) \bar{F}_{X^{f}}(x)}{\bar{F}_{X^{m}, X^{f}}(x, x) \bar{F}_{Z}(x) \bar{F}_{X^{f}}(y) \bar{F}_{X^{f}}(x)}= \\
& =\frac{\bar{F}_{X^{m}, X^{f}}(x, y) \bar{F}_{X^{f}}(x)}{\bar{F}_{X^{m}, X^{f}}(x, x) \bar{F}_{X^{f}}(y)} P\left(T^{f}>y \mid T^{f}>x\right)= \\
& =A(x, y) P\left(T^{f}>y \mid T^{f}>x\right) .
\end{aligned}
$$


We will show now the equivalencies.

$(7) \Leftrightarrow(8)$ : Let $(7)$ be true. Observe that $A(x, y)=\frac{\bar{F}_{X^{m}, X^{f}}(x, y) \bar{F}_{X^{f}}(x)}{\bar{F}_{X^{m}, X^{f}}(x, x) \bar{F}_{X^{f}}(y)} \leq 1$, if and only if

$$
\frac{\bar{F}_{X^{m}, X^{f}}(x, y)}{\bar{F}_{X^{m}, X^{f}}(x, x)} \leq \frac{\bar{F}_{X^{f}}(y)}{\bar{F}_{X^{f}}(x)},
$$

which is equivalent to the inequality (8).

$(7) \Leftrightarrow(9)$ : By analogy, $A(x, y) \leq 1$, if and only if

$$
\frac{\bar{F}_{X^{m}, X^{f}}(x, y)}{\bar{F}_{X^{f}}(y)} \leq \frac{\bar{F}_{X^{m}, X^{f}}(x, x)}{\bar{F}_{X^{f}}(x)},
$$

that is, if and only if (9) is valid.

Obviously, analogous relations hold in the case when $x>y$ and when $T^{f}$ and $T^{m}$ are interchanged.

Since if $X^{m}$ and $X^{f}$ are independent random variables then $A(x, y)=1$, inequalities in Proposition 2.1 turn to equalities under the Generalized Marshall-Olkin model and relation (6) holds.

Remark 2.2. Notice that, if $X^{f}$ is right-tail decreasing in $X^{m}$ (that implies negative quadrant dependence), then inequality (9) is fulfilled.

\subsection{Copula version of the EMO model and its properties}

We will assume hereafter that the distributions of the random variables $X^{m}, X^{f}$ and $Z$ involved in (1) are absolutely continuous with strictly decreasing survival distribution functions on $[0,+\infty)$.

We rewrite the EMO model (3) as

$$
\bar{F}_{T^{m}, T^{f}}(x, y)=C\left(\bar{F}_{X^{m}}(x), \bar{F}_{X^{f}}(y)\right) \bar{F}_{Z}(\max (x, y)),
$$

where $C$ is an absolutely continuous copula connecting the survival marginal distributions of $X^{m}$ and $X^{f}$ from the entry ages $x_{m}$ and $x_{f}$ respectively.

Observe that the joint distribution of $\left(T^{m}, T^{f}\right)$ specified by (10) is not absolutely continuous since the probability of the event $\left\{T^{m}=T^{f}\right\}$ is not negligible. In fact,

$$
\begin{aligned}
P\left(T^{m}=T^{f}>t\right) & =E\left[P\left(Z \leq X^{m}, Z \leq X^{f}, Z>t \mid Z\right)\right]= \\
& =\int_{t}^{+\infty} C\left(\bar{F}_{X^{m}}(z), \bar{F}_{X^{f}}(z)\right) d F_{Z}(z),
\end{aligned}
$$

from which

$$
P\left(T^{m}=T^{f}\right)=\int_{0}^{+\infty} C\left(\bar{F}_{X^{m}}(z), \bar{F}_{X^{f}}(z)\right) d F_{Z}(z)>0 .
$$


The marginal survival distribution functions of $T^{m}$ and $T^{f}$ can be obtained from (3) as follows

$$
\bar{F}_{T^{m}}(x)=\bar{F}_{X^{m}}(x) \bar{F}_{Z}(x) \quad \text { and } \quad \bar{F}_{T^{f}}(x)=\bar{F}_{X^{f}}(x) \bar{F}_{Z}(x), \quad x \geq 0 .
$$

Applying Sklar's theorem in (10) we get the dependence structure between $T^{m}$ and $T^{f}$ expressed by the associated survival copula

$C_{T^{m}, T^{f}}(u, v)=C\left(\bar{F}_{X^{m}} \circ \bar{F}_{T^{m}}^{-1}(u), \bar{F}_{X^{f}} \circ \bar{F}_{T^{f}}^{-1}(v)\right) \bar{F}_{Z}\left(\max \left(\bar{F}_{T^{m}}^{-1}(u), \bar{F}_{T^{f}}^{-1}(v)\right)\right), u, v \in[0,1]$,

where $\circ$ is the composition operator. Thanks to (12), we have

$$
\bar{F}_{X^{m}} \circ \bar{F}_{T^{m}}^{-1}(u)=\frac{u}{\bar{F}_{Z} \circ \bar{F}_{T^{m}}^{-1}(u)} \quad \text { and } \quad \bar{F}_{X^{f}} \circ \bar{F}_{T^{f}}^{-1}(u)=\frac{u}{\bar{F}_{Z} \circ \bar{F}_{T^{f}}^{-1}(u)},
$$

yielding

$$
C_{T^{m}, T^{f}}(u, v)=C\left(\frac{u}{\bar{F}_{Z} \circ \bar{F}_{T^{m}}^{-1}(u)}, \frac{v}{\bar{F}_{Z} \circ \bar{F}_{T^{f}}^{-1}(v)}\right) \min \left(\bar{F}_{Z} \circ \bar{F}_{T^{m}}^{-1}(u), \bar{F}_{Z} \circ \bar{F}_{T^{f}}^{-1}(v)\right),
$$

which will be referred as EMO copula. By construction, the copula $C_{T^{m}, T^{f}}$ is not absolutely continuous since the singularity of the joint distribution of $\left(T^{m}, T^{f}\right)$ induces a singularity on the set

$$
\mathcal{S}=\left\{(u, v) \in[0,1]^{2}: v=g(u)=\bar{F}_{T^{f}} \circ \bar{F}_{T^{m}}^{-1}(u)\right\} .
$$

As we noted earlier, in Denuit et al. (2006) the authors assume that $X^{m}$ and $X^{f}$ are independent random variables in the stochastic representation (3). As a consequence of their Proposition 4.1, the authors deduce that under the Generalized Marshall-Olkin model, increasing dependence between $T^{m}$ and $T^{f}$ can be achieved by increasing the force of mortality of the common shock $r_{Z}$ and decreasing both individual ones $r_{X^{m}}$ and $r_{X^{f}}$, such that $r_{X^{m}}+r_{Z}$ and $r_{X^{f}}+r_{Z}$ remain fixed. A nice interpretation of this fact was offered by one of the referees as follows: "If the common shock becomes more significant, therefore adding positive dependence to the model, then, with fixed marginal distributions for $T^{m}$ and $T^{f}, X^{m}$ and $X^{f}$ become less significant, and, since these are independent, will in no way compensate for the stronger common effect".

Such a conclusion is not always true if $X^{m}$ and $X^{f}$ are dependent, i.e., when the EMO model is valid. In order to analyze this point, let us consider two random variables $Z$ and $U$ representing the common shocks, such that their survival functions fulfill $\bar{F}_{Z}(x) \geq \bar{F}_{U}(x)$ for all $x \geq 0$. Notice that, the corresponding joint survival distributions for $T^{m}$ and $T^{f}, \bar{F}_{T^{m}, T^{f}}\left(x, y ; \bar{F}_{Z}\right)$ and $\bar{F}_{T^{m}, T^{f}}\left(x, y ; \bar{F}_{U}\right)$ satisfy the relation

$$
\bar{F}_{T^{m}, T^{f}}\left(x, y ; \bar{F}_{Z}\right) \leq \bar{F}_{T^{m}, T^{f}}\left(x, y ; \bar{F}_{U}\right), \quad x, y \geq 0
$$


if and only if, the corresponding copulas $C_{T^{m}, T^{f}}\left(u, v ; \bar{F}_{Z}\right)$ and $C_{T^{m}, T^{f}}\left(u, v ; \bar{F}_{U}\right)$ satisfy the inequality

$$
C_{T^{m}, T^{f}}\left(u, v ; \bar{F}_{Z}\right) \leq C_{T^{m}, T^{f}}\left(u, v ; \bar{F}_{U}\right), \quad u, v \in[0,1] .
$$

Moreover, since the marginal distributions of $T^{m}$ and $T^{f}$ are assumed to be unchanged, the two copulas in (15) share the same singularity curve (see (13)). Under the above notations we will prove the following

Proposition 2.2. Let $C$ be the copula of the vector $\left(X^{m}, X^{f}\right)$. If $\frac{C(u, v)}{u}$ is nondecreasing in $u$ and $\frac{C(u, v)}{v}$ is non-decreasing in $v$ for $u, v \in[0,1]$ and if $\bar{F}_{Z}(x) \geq$ $\bar{F}_{U}(x)$ for all $x \geq 0$, then (14) holds for all $(x, y) \in[0,+\infty)^{2}$.

Proof. Let us consider the case $x>y$. Then (14) rewrites

$$
C\left(\frac{\bar{F}_{T^{m}}(x)}{\bar{F}_{Z}(x)}, \frac{\bar{F}_{T^{f}}(y)}{\bar{F}_{Z}(y)}\right) \bar{F}_{Z}(x) \leq C\left(\frac{\bar{F}_{T^{m}}(x)}{\bar{F}_{U}(x)}, \frac{\bar{F}_{T^{f}}(y)}{\bar{F}_{U}(y)}\right) \bar{F}_{U}(x)
$$

and this is equivalent to

$$
\frac{C\left(\frac{\bar{F}_{T}(x)}{\bar{F}_{Z}(x)}, \frac{\bar{F}_{T f}(y)}{\bar{F}_{Z}(y)}\right)}{\frac{\bar{F}_{T^{m}}(x)}{\bar{F}_{Z}(x)}} \leq \frac{C\left(\frac{\bar{F}_{T^{m}}(x)}{\bar{F}_{U}(x)}, \frac{\bar{F}_{T f}(y)}{\bar{F}_{U}(y)}\right)}{\frac{\bar{F}_{T^{m}}(x)}{\bar{F}_{U}(x)}} .
$$

Thanks to the assumptions we have that

$$
\frac{C\left(\frac{\bar{F}_{T^{m}}(x)}{\bar{F}_{Z}(x)}, \frac{\bar{F}_{T^{f}}(y)}{\bar{F}_{Z}(y)}\right)}{\frac{\bar{F}_{T^{m}}(x)}{\bar{F}_{Z}(x)}} \leq \frac{C\left(\frac{\bar{F}_{T^{m}}(x)}{\bar{F}_{U}(x)}, \frac{\bar{F}_{T^{f}}(y)}{\bar{F}_{Z}(y)}\right)}{\frac{\bar{F}_{T^{m}}(x)}{\bar{F}_{U}(x)}} \leq \frac{C\left(\frac{\bar{F}_{T^{m}}(x)}{\bar{F}_{U}(x)}, \frac{\bar{F}_{T^{f}}(y)}{\bar{F}_{U}(y)}\right)}{\frac{\bar{F}_{T^{m}}(x)}{\bar{F}_{U}(x)}} .
$$

Similar arguments hold in the case $x<y$ and the thesis follows.

Remark 2.3. In fact, the sufficient conditions on the copula $C$ in Proposition 2.2 are equivalent to $X^{f}$ being right tail decreasing in $X^{m}$, and $X^{m}$ being right tail decreasing in $X^{f}$, respectively, both implying negative quadrant dependence of the two random variables (see for more details Section 5.2.2 in Nelsen, 2006).

If the non-decreasing requirements on the copula $C$ in Proposition 2.2. fail to hold, then inequality (14) might be not satisfied for all $x, y \geq 0$. In next example and remarks we discuss this phenomenon.

Example 2.2. Let $\bar{F}_{T^{m}}(x)=e^{-\lambda_{m} x}, \bar{F}_{T^{f}}(x)=e^{-\lambda_{f} x}, \bar{F}_{Z}(x)=e^{-\lambda x}$ and $\bar{F}_{U}(x)=$ $e^{-\mu x}$ with $\lambda_{m}>\lambda_{f}>\mu>\lambda>0$. Applying (10), we have

$$
\bar{F}_{T^{m}, T^{f}}\left(x, y ; \bar{F}_{Z}\right)=C\left(e^{-\left(\lambda_{m}-\lambda\right) x}, e^{-\left(\lambda_{f}-\lambda\right) y}\right) e^{-\lambda \max (x, y)}
$$


and

$$
\bar{F}_{T^{m}, T^{f}}\left(x, y ; \bar{F}_{U}\right)=C\left(e^{-\left(\lambda_{m}-\mu\right) x}, e^{-\left(\lambda_{f}-\mu\right) y}\right) e^{-\mu \max (x, y)} .
$$

Let $C$ be the Clayton copula joining marginals $X^{m}$ and $X^{f}$, i.e.,

$$
C(u, v)=\left(u^{-\theta}+v^{-\theta}-1\right)^{-1 / \theta}, \quad \theta>0, u, v \in[0,1] .
$$

Assume that $x \geq y$. Then (14) holds if and only if

$$
\left(e^{\left(\lambda_{m}-\lambda\right) \theta x}+e^{\left(\lambda_{f}-\lambda\right) \theta y}-1\right)^{-1 / \theta} e^{-\lambda x} \leq\left(e^{\left(\lambda_{m}-\mu\right) \theta x}+e^{\left(\lambda_{f}-\mu\right) \theta y}-1\right)^{-1 / \theta} e^{-\mu x}
$$

from which

$$
\left(e^{\left(\lambda_{m}-\lambda\right) \theta x}+e^{\left(\lambda_{f}-\lambda\right) \theta y}-1\right) e^{\theta \lambda x} \geq\left(e^{\left(\lambda_{m}-\mu\right) \theta x}+e^{\left(\lambda_{f}-\mu\right) \theta y}-1\right) e^{\theta \mu x},
$$

that is

$$
e^{\lambda \theta x}\left(e^{\theta\left(\lambda_{f}-\lambda\right) y}-1\right) \geq e^{\mu \theta x}\left(e^{\theta\left(\lambda_{f}-\mu\right) y}-1\right) .
$$

This means that the inequality (14) is fulfilled only for those pairs $(x, y)$ so that

$$
y \leq x \leq \frac{1}{(\mu-\lambda) \theta} \log \left(\frac{e^{\theta\left(\lambda_{f}-\lambda\right) y}-1}{e^{\theta\left(\lambda_{f}-\mu\right) y}-1}\right) .
$$

A similar restriction holds when $y>x$.

Remark 2.4. There are cases in which inequality (14) is reversed for all $(x, y) \in$ $[0,+\infty)^{2}$. Consider the Example 2.2 and let $\theta$ go to $+\infty$. As a result we obtain that $C(u, v)=\min (u, v)$ (that is, $X^{m}$ and $X^{f}$ are comonotonic random variables) and, since $\frac{1}{(\mu-\lambda) \theta} \log \left(\frac{e^{\theta\left(\lambda_{f}-\lambda\right) y}-1}{e^{\theta\left(\lambda_{f}-\mu\right) y}-1}\right) \rightarrow 0$, we conclude that

$$
\bar{F}_{T^{m}, T^{f}}\left(x, y ; \bar{F}_{Z}\right) \geq \bar{F}_{T^{m}, T^{f}}\left(x, y ; \bar{F}_{U}\right), \quad \text { for all } \quad(x, y) \in[0,+\infty)^{2} .
$$

Remark 2.5. In the specific case when $X^{m}$ and $X^{f}$ are comonotonic random variables with the connecting copula $C(u, v)=\min (u, v)$ and under the assumption that $T^{m}$ and $T^{f}$ share the same exponential distribution with parameter $\bar{\lambda}$ (i.e., when $\lambda_{m}=\lambda_{f}=\bar{\lambda}$ in Example 2.2), from (16) and (17) we get

$$
\bar{F}_{T^{m}, T^{f}}\left(x, y ; \bar{F}_{Z}\right)=e^{-\bar{\lambda} \max (x, y)} \quad \text { and } \quad \bar{F}_{T^{m}, T^{f}}\left(x, y ; \bar{F}_{U}\right)=e^{-\bar{\lambda} \max (x, y)} .
$$

Hence,

$$
\bar{F}_{T^{m}, T^{f}}\left(x, y ; \bar{F}_{Z}\right)=\bar{F}_{T^{m}, T^{f}}\left(x, y ; \bar{F}_{U}\right) .
$$

Moreover, equality (18) is always true if $C(u, v)=\min (u, v)$ and if $T^{m}$ and $T^{f}$ are identically distributed (not necessarily exponential). Indeed, let $\bar{F}_{T^{m}}(x)=$ 
$\bar{F}_{T^{f}}(x)=\bar{H}(x)$ for all $x \geq 0$. In the presence of the common shock arrival time identified by $Z$, we have that $\bar{F}_{X^{m}}(x)=\bar{F}_{X^{f}}(x)=\frac{\bar{H}(x)}{\bar{F}_{Z}(x)}$, and therefore

$$
\bar{F}_{T^{m}, T^{f}}\left(x, y ; \bar{F}_{Z}\right)=\min \left(\frac{\bar{H}(x)}{\bar{F}_{Z}(x)}, \frac{\bar{H}(y)}{\bar{F}_{Z}(y)}\right) \cdot \bar{F}_{Z}(\max (x, y))=\bar{H}(\max (x, y)) .
$$

By analogy, $\bar{F}_{T^{m}, T^{f}}\left(x, y ; \bar{F}_{U}\right)=\bar{H}(\max (x, y))$, and thus (18) is satisfied for all $(x, y) \in[0,+\infty)^{2}$.

One of the referees interpreted this conclusion as follows: "The effect of a higher rate of occurrence of simultaneous death is exactly offset by the effect of a reduced significance of $X^{m}$ and $X^{f}$ ".

\subsection{Mortality intensities relations}

Following Gouriéroux and $\mathrm{Lu}$ (2015) we analyze the behavior of the individual mortality intensities with respect to the occurrence or not of the death of the other individual in the couple.

In order to simplify the notations, given a differentiable function $f(x, y)$, we denote by $\partial_{1} f(x, y)$ and $\partial_{2} f(x, y)$ the two first partial derivatives with respect to the first and the second argument.

The mortality intensity at time $y$ of the male in the couple in the case in which both individuals are alive at time $y$, is defined as

$$
\lambda_{m}\left(y \mid T_{m}>y, T_{f}>y\right)=\lim _{h \rightarrow 0^{+}} \frac{\mathbb{P}\left(y<T_{m} \leq y+h \mid T_{m}>y, T_{f}>y\right)}{h} .
$$

But

$$
\begin{aligned}
\lim _{h \rightarrow 0^{+}} \frac{\mathbb{P}\left(y<T_{m} \leq y+h \mid T_{m}>y, T_{f}>y\right)}{h} & =\frac{1}{\bar{F}_{T^{m}, T^{f}}(y, y)} \lim _{h \rightarrow 0^{+}} \frac{\bar{F}_{T^{m}, T^{f}}(y+h, y)-\bar{F}_{T^{m}, T^{f}}(y, y)}{h}= \\
& =\frac{-\partial_{1} \bar{F}_{X^{m}, X^{f}}(y, y) \bar{F}_{Z}(y)+\bar{F}_{X^{m}, X^{f}}(y, y) f_{Z}(y)}{\bar{F}_{T^{m}, T^{f}}(y, y)}
\end{aligned}
$$

from which

$$
\lambda_{m}\left(y \mid T_{m}>y, T_{f}>y\right)=\frac{-\partial_{1} \bar{F}_{X^{m}, X^{f}}(y, y)}{\bar{F}_{X^{m}, X^{f}}(y, y)}+\frac{f_{Z}(y)}{\bar{F}_{Z}(y)} .
$$

Notice that the mortality intensity at time $y$ of the male in the couple, in the case in which both individuals are alive at time $y$, turns out to be the sum of the hazard rate in $y$ of $X^{m}$ given that $X^{f}>y$ and the hazard rate of $Z$ in $y$.

The corresponding intensity for the female is defined and obtained similarly:

$$
\lambda_{f}\left(y \mid T_{m}>y, T_{f}>y\right)=\frac{-\partial_{2} \bar{F}_{X^{m}, X^{f}}(y, y)}{\bar{F}_{X^{m}, X^{f}}(y, y)}+\frac{f_{Z}(y)}{\bar{F}_{Z}(y)} .
$$


On the other side, the mortality intensity at time $y$ of the male in the couple in the case in which his wife dies at time $y$, is defined as

$$
\lambda_{m \mid f}\left(y \mid T_{m}>y, T_{f}=y\right)=\lim _{h \rightarrow 0^{+}} \frac{\mathbb{P}\left(y<T_{m} \leq y+h \mid T_{m}>y, T_{f}=y\right)}{h} .
$$

But, since, for $x>y$

$$
\mathbb{P}\left(T^{m}>x \mid T_{f}=y\right)=\partial_{2} \bar{F}_{X^{m}, X^{f}}(x, y) \bar{F}_{Z}(x) / \bar{F}_{T^{f}}^{\prime}(y),
$$

$$
\begin{aligned}
\lim _{h \rightarrow 0^{+}} \frac{\mathbb{P}\left(y<T_{m} \leq y+h \mid T_{m}>y, T_{f}=y\right)}{h} & =-\frac{\lim _{h \rightarrow 0^{+}} \frac{\partial_{2} \bar{F}_{X^{m}, X}(y+h, y) \bar{F}_{Z}(y+h)-\partial_{2} \bar{F}_{X^{m}, X f}(y, y) \bar{F}_{Z}(y)}{h}}{\partial_{2} \bar{F}_{X^{m}, X^{f}}(y, y) \bar{F}_{Z}(y)}= \\
& =\frac{-\partial_{2} \bar{F}_{X^{m}, X^{f}}(y, y) f_{Z}(y)+f_{X^{m}, X^{f}}(y, y) \bar{F}_{Z}(y)}{-\partial_{2} \bar{F}_{X^{m}, X^{f}}(y, y) \bar{F}_{Z}(y)}
\end{aligned}
$$

and

$$
\lambda_{m \mid f}\left(y \mid T_{m}>y, T_{f}=y\right)=\frac{f_{Z}(y)}{\bar{F}_{Z}(y)}+\frac{f_{X^{m}, X^{f}}(y, y)}{-\partial_{2} \bar{F}_{X^{m}, X^{f}}(y, y)}
$$

that is, the mortality intensity at time $y$ of the male in the couple in the case in which his wife dies at time $y$ is the sum of the hazard rate in $y$ of $X^{m}$ given that $X^{f}=y$ and the hazard rate of $Z$ in $y$.

Similarly, the corresponding intensity for the female is

$$
\lambda_{f \mid m}\left(y \mid T_{m}>y, T_{f}=y\right)=\frac{f_{Z}(y)}{\bar{F}_{Z}(y)}+\frac{f_{X^{m}, X^{f}}(y, y)}{-\partial_{1} \bar{F}_{X^{m}, X^{f}}(y, y)} .
$$

As proposed in Gouriéroux and $\mathrm{Lu}$ (2015), the ratios

$$
\gamma_{m \mid f}(y)=\frac{\lambda_{m \mid f}\left(y \mid T_{m}>y, T_{f}=y\right)}{\lambda_{m}\left(y \mid T_{m}>y, T_{f}>y\right)}=\frac{\frac{f_{X^{m}, X^{f}}(y, y)}{-\partial_{2} \bar{F}_{X^{m}, X^{f}}(y, y)}+\frac{f_{Z}(y)}{\bar{F}_{Z}(y)}}{\frac{-\partial_{1} \bar{F}_{X^{m}, X^{f}}(y, y)}{\bar{F}_{X^{m}, X^{f}}(y, y)}+\frac{f_{Z}(y)}{\bar{F}_{Z}(y)}}
$$

and

$$
\gamma_{f \mid m}(y)=\frac{\lambda_{f \mid m}\left(y \mid T_{m}>y, T_{f}=y\right)}{\lambda_{f}\left(y \mid T_{m}>y, T_{f}>y\right)}=\frac{\frac{f_{X^{m}, X^{f}}(y, y)}{-\partial_{1} \bar{F}_{X^{m}, X^{f}}(y, y)}+\frac{f_{Z}(y)}{\bar{F}_{Z}(y)}}{\frac{-\partial_{2} \bar{F}_{X^{m}, X^{f}}(y, y)}{\bar{F}_{X^{m}, X^{f}}(y, y)}+\frac{f_{Z}(y)}{\bar{F}_{Z}(y)}}
$$

represent a measure of the impact of the wife (husband) death on the husband (wife) mortality intensity. In particular, the case $\gamma_{m \mid f}(y)=1\left(\gamma_{f \mid m}(y)=1\right)$ corresponds to no impact. 
In order to simplify the notation we set

$$
\theta(x, y)=\frac{\bar{F}_{X^{m}, X^{f}}(x, y) f_{X^{m}, X^{f}}(x, y)}{\partial_{1} \bar{F}_{X^{m}, X^{f}}(x, y) \partial_{2} \bar{F}_{X^{m}, X^{f}}(x, y)} .
$$

Through a straightforward computation, from (19) and (20), we have that

$$
\gamma_{m \mid f}(y)=1 \Leftrightarrow \gamma_{f \mid m}(y)=1 \Leftrightarrow \theta(y, y)=1 .
$$

Apart from the case of no impact, the mortality intensities of two individuals in the couple can react in the same way to the death of the other spouse, which corresponds to $\gamma_{m \mid f}(y)=\gamma_{f \mid m}(y) \neq 1$. This is always the case when $\frac{f_{Z}(y)}{\bar{F}_{Z}(y)}=0$ that is when no external catastrophic event is considered, while in the general case when $\frac{f_{Z}(y)}{\bar{F}_{Z}(y)}>0$, it is trivial to check that

$$
\gamma_{m \mid f}(y)=\gamma_{f \mid m}(y) \neq 1 \Leftrightarrow \partial_{1} \bar{F}_{X^{m}, X^{f}}(y, y)=\partial_{2} \bar{F}_{X^{m}, X^{f}}(y, y) .
$$

Conversely, the case in which the male in the couple is more affected by the death of his wife than the female by the death of her husband corresponds to $\gamma_{m \mid f}(y)>$ $\gamma_{f \mid m}(y)$ and we have that

$$
\begin{aligned}
\gamma_{m \mid f}(y)>\gamma_{f \mid m}(y) & \Leftrightarrow \partial_{1} \bar{F}_{X^{m}, X^{f}}(y, y)<\partial_{2} \bar{F}_{X^{m}, X^{f}}(y, y) \text { and } \theta(y, y)>1 \\
& \text { or } \partial_{1} \bar{F}_{X^{m}, X^{f}}(y, y)>\partial_{2} \bar{F}_{X^{m}, X^{f}}(y, y) \text { and } \theta(y, y)<1 .
\end{aligned}
$$

Clearly, the opposite situation, that is $\gamma_{m \mid f}(y)<\gamma_{m \mid f}(y)$ holds in the complementary cases.

Remark 2.6. Notice that all the above listed relations only depend on the joint distribution $\bar{F}_{X^{m}, X^{f}}$. It can be easily checked that in the case in which $X^{m}$ and $X^{f}$ are independent (which corresponds to the Generalized Marshall-Olkin distribution of Li and Pellerey, 2011) the model satisfies condition (22) that is the mortality intensities are not sensitive to the mortality status of the other spouse in the couple.

Remark 2.7. In Gouriéroux and Lu (2015), the ratios $\gamma_{m \mid f}$ and $\gamma_{f \mid m}$ are interpreted as measures of the broken-heart effect. This is not the case in our model, in fact, as shown by (19) and (20), they also depend on the rate of the arrival time of some external catastrophic event that is of course not linked to the broken-heart effect. Clearly, in the EMO model, the broken-heart effect modeling is the one considered in the absolutely continuous part of the distribution.

Remark 2.8. It is worthwhile mentioning that the ratio $\theta(x, y)$ in (21) was already introduced in Oakes (1989) in the study of the dependence introduced by frailties. We remark that, in our EMO model, the function $\theta(\cdot, \cdot)$ only depends on the underlying copula function $C$ and that, by integration, it can be easily verified that

$$
\theta(x, y) \geq 1 \Rightarrow C(u, v) \geq u v
$$

that is $\theta(x, y) \geq 1$ implies that $X^{m}$ and $X^{f}$ are positively quadrant dependent. 


\section{Empirical Analysis}

In this section we will apply the Extended Marshall-Olkin distribution to a sample of censored residual lifetimes of couples of insureds extracted from a dataset of annuities contracts of a large Canadian life insurance company ${ }^{1}$. This dataset has already been analyzed in many papers: see Frees et al. (1996), Carriere (2000), Youn and Shemyakin (1999), Shemyakin and Youn (2006), Ji et al. (2011), among the others.

Since the interval of the entry ages of the insured considered in the dataset is very wide, we will concentrate our analysis on the subsample made of couples of individuals whose minimum entry age is 60. After having additionally removed data corresponding to contracts involving insureds of the same sex and multiple contracts on the same couple, the resulting dataset contains information about 9535 married couples during an observation period of five years from December 29, 1988 to December 31, 1993. The dataset is both left and right censored. It is left truncated as the annuitant information are recorded only from the date they enter the study: this means that insureds who have died before the beginning of the observation period were not taken into account in the study. On the other hand, the dataset is right censored in the sense that most of the insureds were alive at the end of the observation period. From each couple we can draw four different information: the entry ages $x_{m}$ and $x_{f}$ for the male and the female, respectively, and the corresponding censored residual lifetimes under the observation period, $\hat{t}_{m}$ and $\hat{t}_{f}$.

\subsection{Estimation procedure}

As done in Dufresne et al. (2018) for the same dataset, we will apply the twostage parametric method for censored data introduced in Shih and Louis (1995).

Differently from the setup of those papers, our survival joint distribution is not absolutely continuous with respect to the Lebesgue measure in $\mathbb{R}^{2}$. However, it results to be absolutely continuous with respect to a measure dominating the Lebesgue one and having the same singularity straight line (for a formal and detailed study of the method in the case of multivariate Pareto distributions, we refer the reader to Asimit et al., 2016). This fact allows to implement the maximum-likelihood estimation technique to the density with respect to this dominating measure as already done in literature in the non censored data case for many extensions of the classical Marshall-Olkin distribution (see Karlis, 2003, Kundu and Dey, 2009, Kundu and Gupta, 2013, Asimit et al., 2016) and also for the EMO distribution in Pinto and Kolev (2015). However, because of the large amount of the parameters to be estimated, we will consider (unlike the mentioned cases in which the maximum-

\footnotetext{
${ }^{1}$ We wish to thank the Society of Actuaries, through the courtesy of Edward (Jed) Frees and Valdez, for allowing the use of the data in this paper.
} 
likelihood estimation is conducted in one step for all parameters) the two-stage approach for censored data of Shih and Louis (1995), using the density with respect to the dominating measure instead of that with respect the Lebesgue measure.

More precisely, according to Asimit et al. (2016) we consider the dominating measure $\mu$ on the plane defined, for every Borel set $B$ of the plane as

$$
\mu(B)=\mathcal{L}_{2}(B)+\mathcal{L}_{1}(s(B))
$$

where $\mathcal{L}_{2}$ and $\mathcal{L}_{1}$ denote the Lebesgue measures on the plane and on the straight line, respectively, and

$$
s(B)=\{x \in \mathbb{R}:(t, s) \in B, t=s=x\} .
$$

The density of the Extended Marshall-Olkin distribution with respect to $\mu, f_{T^{m}, T^{f}}$, is given in Lemma 6.3 in Pinto and Kolev (2015) and in terms of our notation can be rewritten as

$$
f_{T^{m}, T^{f}}(t, s)=\left\{\begin{array}{cc}
f_{X^{m}, X^{f}}(t, s) \bar{F}_{Z}(t)+\partial_{2} \bar{F}_{X^{m}, X^{f}}(t, s) f_{Z}(t), & t>s \geq 0 \\
f_{X^{m}, X^{f}}(t, s) \bar{F}_{Z}(s)+\partial_{1} \bar{F}_{X^{m}, X^{f}}(t, s) f_{Z}(s), & 0 \leq t<s \\
C\left(\bar{F}_{X^{m}}(x), \bar{F}_{X^{f}}(x)\right) f_{Z}(x), & t=s=x \geq 0
\end{array} .\right.
$$

We assume that the involved marginal distributions as well as the underlying copula function $C$ belong to some parametric families: let $\boldsymbol{\theta}^{j}$ be the vector of parameters of the distribution of $T^{j}$, for $j=m, f$, and $\boldsymbol{\alpha}$ be the vector of the remaining dependence parameters.

Let now consider a sample of $i=1, \ldots, n$ censored observed residual lifetimes pairs. According to Shih and Louis (1995), if $\left(C_{i}^{m}, C_{i}^{f}\right)$ denote independent random censoring times for the male and the female individuals in the couple $i$, observations $\left(\hat{\boldsymbol{t}}^{m}, \hat{\boldsymbol{t}}^{f}\right)=\left\{\left(\hat{t}_{i}^{m}, \hat{t}_{i}^{f}\right): i=1, \ldots, n\right\}$ are defined as

$$
\hat{t}_{i}^{m}=\min \left(t_{i}^{m}, C_{i}^{m}\right) \text { and } \hat{t}_{i}^{f}=\min \left(t_{i}^{f}, C_{i}^{f}\right), i=1, \ldots, n
$$

where $t_{i}^{m}$ and $t_{i}^{f}$ are the residual lifetimes. If $\delta_{i, j}=\mathbf{1}_{\left\{\hat{t}_{i}^{j}=t_{i}^{j}\right\}}$ for $i=1, \ldots, n$ and $j=m, f$, the likelihood of the vector of parameters $\boldsymbol{\theta}=\left(\boldsymbol{\theta}^{m}, \boldsymbol{\theta}^{f}, \boldsymbol{\alpha}\right)$ is

$$
\begin{aligned}
L\left(\left(\hat{\boldsymbol{t}}^{m}, \hat{\boldsymbol{t}}^{f}\right) ; \boldsymbol{\theta}\right) & =\prod_{i=1}^{n}\left[f_{T^{m}, T^{f}}\left(t_{i}^{m}, t_{i}^{f} ; \boldsymbol{\theta}\right)\right]^{\delta_{i, m} \delta_{i, f}} \cdot\left[-\partial_{1} \bar{F}_{T^{m}, T^{f}}\left(t_{i}^{m}, C_{i}^{f} ; \boldsymbol{\theta}\right)\right]^{\delta_{i, m}\left(1-\delta_{i, f}\right)} \cdot \\
& \cdot\left[-\partial_{2} \bar{F}_{T^{m}, T^{f}}\left(C_{i}^{m}, t_{i}^{f} ; \boldsymbol{\theta}\right)\right]^{\left(1-\delta_{i, m}\right) \delta_{i, f}} \cdot\left[\bar{F}_{T^{m}, T^{f}}\left(C_{i}^{m}, C_{i}^{f} ; \boldsymbol{\theta}\right)\right]^{\left(1-\delta_{i, m}\right)\left(1-\delta_{i, f}\right)}
\end{aligned}
$$

The two-stage parametric estimation consists in 
1. estimating $\left(\boldsymbol{\theta}^{m}, \boldsymbol{\theta}^{f}\right)$ applying the maximum likelihood estimation technique separately to the marginal distributions (which correspond to assuming independence in the likelihood (23));

2. given the so obtained estimates $\left(\hat{\boldsymbol{\theta}}^{m}, \hat{\boldsymbol{\theta}}^{f}\right)$, find the estimator $\hat{\boldsymbol{\alpha}}$ of $\boldsymbol{\alpha}$, by solving

$$
\underset{\boldsymbol{\alpha}}{\operatorname{argmax}} L\left(\left(\hat{\boldsymbol{t}}^{m}, \hat{\boldsymbol{t}}^{f}\right) ;\left(\hat{\boldsymbol{\theta}}^{m}, \hat{\boldsymbol{\theta}}^{f}, \boldsymbol{\alpha}\right)\right) \text {. }
$$

\subsection{The joint lifetimes model specification}

In actuarial literature many families of distributions have been proposed and studied to fit the distributional properties of residual lifetimes. Taking into account the dataset on which we want to test the EMO model, we assume that marginal residual lifetimes follow a Gompertz law, taking into account the fact that in Frees et al (1996) and in Carriere (2000) it was proved that this distribution performed a very good fit in this dataset (the same choice is done in Dufresne et al., 2018, and in a generalized approach in Luciano et al., 2008 and 2016, and Ji et al, 2011). More precisely, we assume that residual lifetimes survival distributions from ages $x^{m}$ and $x^{f}$ are given by

$$
\bar{F}_{T^{m}}(t)=\exp \left(a_{m}\left(1-e^{\frac{t}{\sigma_{m}}}\right)\right) \text { and } \bar{F}_{T^{f}}(t)=\exp \left(a_{f}\left(1-e^{\frac{t}{\sigma_{f}}}\right)\right)
$$

with $a_{m}=\exp \left(\frac{x_{m}-M_{m}}{\sigma_{m}}\right)$ and $a_{f}=\exp \left(\frac{x_{f}-M_{f}}{\sigma_{f}}\right)$, where $M_{i}$ and $\sigma_{i}$, for $i=m, f$ are the corresponding mode and dispersion parameters of the unconditional distributions.

As for the external independent shock arrival time $Z$, we assume that it is exponentially distributed with parameter $\lambda=\lambda\left(x_{m}, x_{f}\right)$. Since from (12) it follows that

$$
\bar{F}_{T^{m}}(t) \leq \min \left(\bar{F}_{X^{m}}(t), \bar{F}_{Z}(t)\right) \text { and } \bar{F}_{T^{f}}(t) \leq \min \left(\bar{F}_{X^{f}}(t), \bar{F}_{Z}(t)\right),
$$

then, necessarily

$$
\exp \left(a_{m}\left(1-e^{\frac{t}{\sigma_{m}}}\right)\right) \leq e^{-\lambda t} \text { and } \bar{F}_{T^{f}}(t)=\exp \left(a_{f}\left(1-e^{\frac{t}{\sigma_{f}}}\right)\right) \leq e^{-\lambda t}
$$

for all $t \geq 0$ and this condition is satisfied if and only if

$$
\lambda \leq \min \left(\frac{a_{m}}{\sigma_{m}}, \frac{a_{f}}{\sigma_{f}}\right) .
$$

Thanks to (10) and (12), the joint survival distribution function of $\left(T^{m}, T^{f}\right)$ is

$$
\bar{F}_{T^{m}, T^{f}}(t, s)=C\left(e^{a_{m}\left(1-e^{\frac{t}{\sigma_{m}}}\right)+\lambda t}, e^{a_{f}\left(1-e^{\frac{t}{\sigma_{f}}}\right)+\lambda t}\right) e^{-\lambda \max (t, s)} .
$$




\begin{tabular}{|c|c|c|c|}
\hline$\hat{M}_{m}$ & $\hat{M}_{f}$ & $\hat{\sigma}_{m}$ & $\hat{\sigma}_{f}$ \\
\hline 86.1143493 & 92.036869 & 9.564189 & 7.819468 \\
\hline
\end{tabular}

Table 1: Maximum likelihood estimators of the parameters of the marginal distributions.

\begin{tabular}{|ccc|ccc|ccc|cc|}
\hline \multicolumn{3}{|c|}{ Clayton } & \multicolumn{3}{c|}{ Gumbel } & \multicolumn{3}{c|}{ Frank } & \multicolumn{2}{l|}{ Ind } \\
\hline$\hat{\alpha}$ & $\hat{\lambda}(\%)$ & BIC & $\hat{\alpha}$ & $\hat{\lambda}(\%)$ & BIC & $\hat{\alpha}$ & $\hat{\lambda}(\%)$ & BIC & $\hat{\lambda}(\%)$ & BIC \\
\hline 1.1678 & 0.1178 & 3039.03 & 1.0651 & 0.1255 & 3046.478 & 2.2518 & 0.1096 & 3035.478 & 0.1368 & 3091.377 \\
$(0.0047)$ & $(0.0000)$ & & $(0.0001)$ & $(0.0000)$ & & $(0.0107)$ & $(0.0000)$ & & $(0.0000)$ & \\
\hline
\end{tabular}

Table 2: Maximum likelihood estimators and relative standard errors. Copula and exponential parameters are assumed to be constant across the dataset. The order of magnitude of standard errors is lower than $5 \times 10^{-8}$.

\subsection{Empirical results}

As described in subsection 3.1, at stage one, we compute the maximum-likelihood estimators of the parameters of the marginal Gompertz distributions. The estimated parameters are listed in Table 1.

At stage two, we estimate the intensity of $Z, \lambda$, and the parameter $\alpha$ of the considered copula family: in the analysis both parameters are assumed to be constant that is independent of the entry ages. As copulas families, we consider the Clayton, the Frank and the Gumbel ones. Moreover, in order to compare the Extended Marshall-Olkin model to the classical Generalized Marshall-Olkin model, we also consider the product copula, that is the independence case.

As done in Ji et al. (2011), in order to take into account a delay in reporting the exact date of death, we consider as simultaneous deaths (due to catastrophic events) those occurring by a 5 days lag. Because of (24), given the estimated parameters of the marginal distributions, the constant intensity $\lambda$ of the occurrence of a catastrophic event will be constrained to be lower than $\lambda_{\max }=0.2126 \%$. The obtained maximum-likelihood estimators are showed in Table 2.

In order to compare the considered copula models we calculate the Bayesian Information Criterion, modified in order to take into account the presence of censored data as suggested in Volinsky and Raftery (2000), that is

$$
B I C=-2 \cdot L L+k \cdot \log r
$$

where $L L$ is the maximum value of the log-likelihood, $k$ is the number of parameters to be estimated and $r$ is the number of censored data.

Comparing BIC values, the best performance is achieved by the Frank copula with parameter 2.2518 and external shock intensity parameter $0.1096 \%$. 

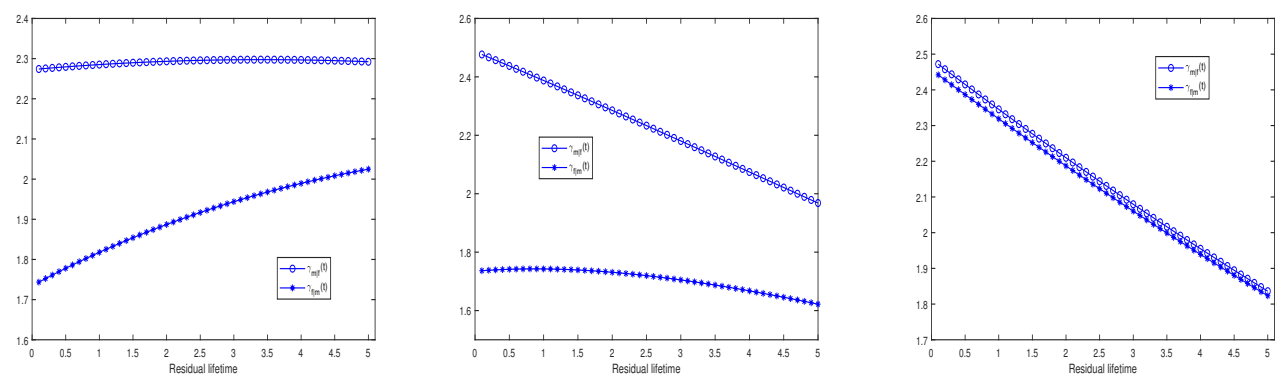

Figure 1: Dynamics of mortality intensities ratios $\gamma_{m \mid f}$ and $\gamma_{f \mid m}$ under the previously estimated model. Left: $x_{m}=x_{f}=60$; middle: $x_{m}=80, x_{f}=60$; right: $x_{m}=x_{f}=$ 80 .

In Figure 1, under the estimated model (that is marginal distributions of Gompertz type with parameters in Table 1, Frank copula with parameter $\alpha=2.2518$ and external catastrophic event with arrival intensity $\lambda=0.1096 \%$ ), we compare the dynamics of the mortality intensities ratios in (19) and (20) during the observation period. The cases of younger and older spouses with the same ages (60-60 and 8080 ) and the case of a large age difference with the husband 20 years older than his wife, are considered. It can be clearly observed that in any case the male mortality intensity is much more increased by the occurrence of the wife death than vice versa and the difference reduces when both are old. We remind that these coefficients are the results of the compounded effects of the broken-heart effect and the possibility of the occurrence of a catastrophic event causing the death of the widower and that (see Remark 2.7) the differences between the two ratios are due to the assumption of the possible occurrence of the external fatal event.

\section{Insurance pricing}

In what follows we will analyze some very common joint life insurance products under the EMO model. For the sake of simplicity, we will assume a constant interest rate intensity $r>0$.

\section{Continuous $n$-years joint life annuity}

This contract pays $1 \$$ per year as long as both spouses survive up to a term $n$. The net premium is given in equation (2)

$$
\bar{a}_{x y ; n\rceil}=\int_{0}^{n} e^{-r t} \bar{F}_{T^{m}, T^{f}}(t, t) d t=\int_{0}^{n} e^{-r t} \bar{F}_{X^{m}, X^{f}}(t, t) \bar{F}_{Z}(t) d t .
$$

Since the dependence structure of $T^{m}$ and $T^{f}$ depends on the underlying copula $C$ 
and on the hazard rate of $Z$, let us analyze the effect of the two on the annuity premium.

If $C_{1} \succ C_{2}$, meaning that $C_{1}(u, v) \geq C_{2}(u, v)$, then, if

$$
\bar{F}_{T^{m}, T^{f}}^{(i)}(t, s)=C_{i}\left(\bar{F}_{X^{m}}(t), \bar{F}_{X^{f}}(s)\right) \bar{F}_{Z}(\max (t, s)), \text { for } i=1,2,
$$

it follows that

$$
\bar{a}_{x y ; n\rceil}^{(1)}=\int_{0}^{n} e^{-r t} \bar{F}_{T^{m}, T^{f}}^{(1)}(t, t) d t \geq \int_{t=0}^{n} e^{-r t} \bar{F}_{T^{m}, T^{f}}^{(2)}(t, t) d t=\bar{a}_{x y ; n\rceil}^{(2)} .
$$

- If the relationship between a husband and his wife is good, then the possible bivariate model accounting for the positive dependence between spouses' lifetimes should be based on positive quadrant dependence property. In such a case $C(u, v) \geq u v$ and the corresponding net single premium $\bar{a}_{x y ; n\rceil}^{E M O}>\bar{a}_{x y ; n\rceil}^{G M O}$. Therefore, if the common lifestyle is comfortable for a couple, then the EMO model is more conservative, indicating a higher net single premium in comparison to model (1).

- If $C(u, v)<u v$, the net single premium $\bar{a}_{x y ; n\rceil}^{E M O}$ is less than $\bar{a}_{x y ; \bar{n} \mid}^{G M O}$. This is the case when the relationship between spouses (between $X^{m}$ and $X^{f}$ ) is negative. For example, let $\left(X^{m}, X^{f}\right)$ be distributed according to the Gumbel's type I bivariate exponential distribution as in (4) which is negative quadrant dependent. So, in the case of negative quadrant dependence, pricing using the Generalized Marshall-Olkin model, implies an overestimation of the premium.

As observed in Section 2 (more specifically in Proposition 2.2, Example 2.2 and Remarks 2.4 and 2.5) the effect on the joint survival distribution (and consequently on the joint life annuity premium) of different hazard rates of $Z$ (keeping the marginal distributions of $T^{m}$ and $T^{f}$ unchanged) is less immediate.

In Figure 2 we analyze the behavior of the net single premium of a term continuous annuity with term $n=5$ years with respect to different values of the intensity of $Z$ (that is assumed to be exponentially distributed with intensity $\lambda$ ), different families of Archimedean copulas $C$ and different levels of dependence (measured through the Kendall's tau parameter). The distributions of the residual lifetimes of both individuals in the couple are of Gompertz type with the parameters estimated in the previous section and showed in Table 1 and the pairs of entry ages analyzed are again 60-60, 80-60 and 80-80. In all entry ages cases, the copula that gives the greatest premium is the Gumbel one: this is clearly due to the fact that the Gumbel copula exhibits positive upper tail dependence. Moreover, in all cases, as the value of $\lambda$ increases, the impact of the different copulas become less relevant. This is due to the fact that, as $\lambda$ increases, since the marginal distributions of $T^{m}$ and 
$T^{f}$ remain unchanged, $\bar{F}_{X^{m}}$ and $\bar{F}_{X^{f}}$ must increase (according to (12)) until, for $\lambda$ sufficiently large and consistent with the assumed marginal distributions of $T^{m}$ and $T^{f}$ (see (24)), they are very close to 1 and so the impact of different copula types reduces. However, in all cases, we observe an increase in the premium value with $\lambda$.
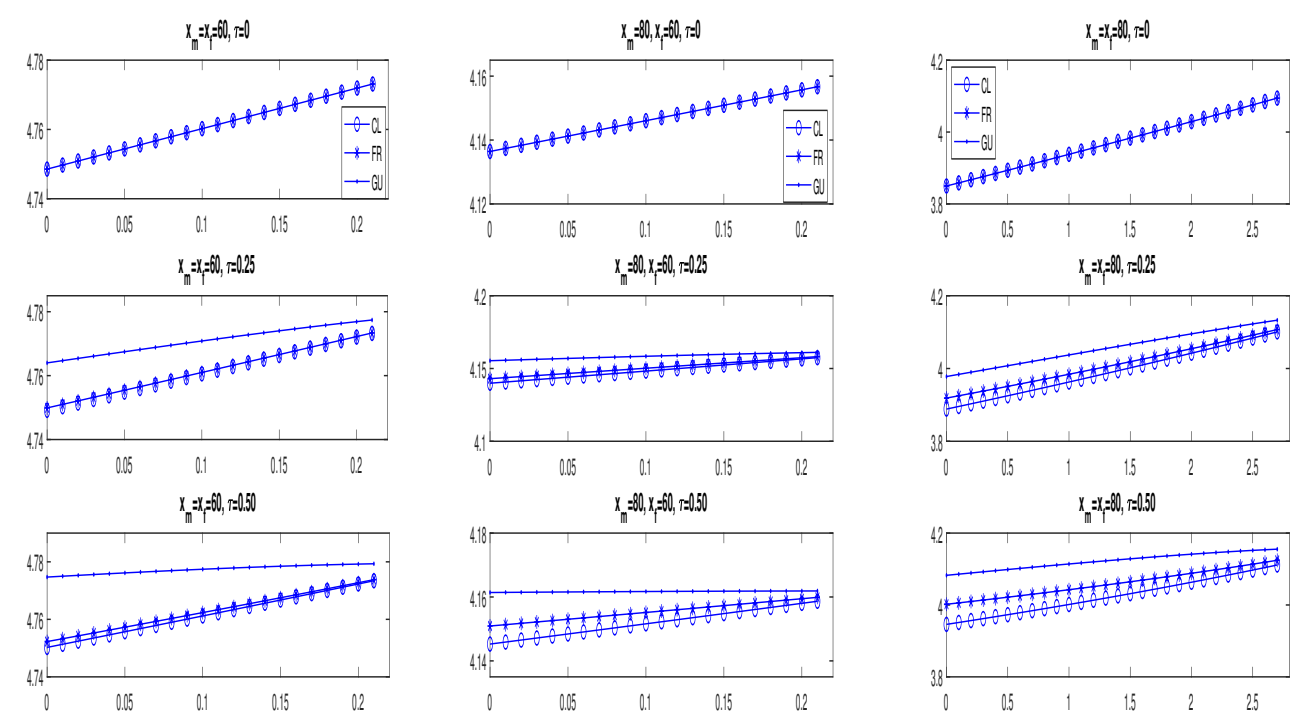

Figure 2: Continuous 5-year joint life annuity premiums by different entry ages. The premiums dynamics are analyzed with respect to the external fatal shock intensity $\lambda$ (on the horizontal axis, expressed in percentages), with respect to different underlying copulas and with respect to different levels of dependence given by different Kendall's tau coefficients. The instantaneous rate of interest is $r=0.01$. Left: $x_{m}=x_{f}=60$; middle: $x_{m}=80, x_{f}=60$; right: $x_{m}=x_{f}=80$.

\section{Joint life, last survivor and simultaneous death policies}

A joint life policy pays a fixed lump sum on the first death between the spouses. The expected present value of the future cash-flow (in case of a unitary lump sum) is

$$
\bar{A}_{x_{m} x_{f}}^{(1)}=\mathbb{E}\left[e^{-r \min \left(T^{m}, T^{f}\right)}\right]=1-r \int_{0}^{+\infty} e^{-r y} \bar{F}_{T^{m}, T^{f}}(y, y) d y .
$$

As for the last survivor unitary policy, the lump sum is paid on the second of the two deaths. The expected present value is

$$
\bar{A}_{x_{m} x_{f}}^{(2)}=\mathbb{E}\left[e^{-r \max \left(T^{m}, T^{f}\right)}\right]=\bar{A}_{x_{m}}+\bar{A}_{x_{f}}-\bar{A}_{x_{m} x_{f}}
$$


where $\bar{A}_{x_{m}}$ and $\bar{A}_{x^{f}}$ are the individual whole life insurance policies.

In Figure 3 we analyze the behavior, with respect to the fatal event intensity $\lambda$, of the expected present value of a last survival policy where the marginal distributions are those estimated from the considered sample. The analysis is again conducted varying the copulas and the level of dependence. Again the copula that induces higher prices (whatever is the level of dependence) is the Gumbel copula but the impact of a different copula is almost irrelevant with very old couples. In any case the expected present value increases with the level of dependence.
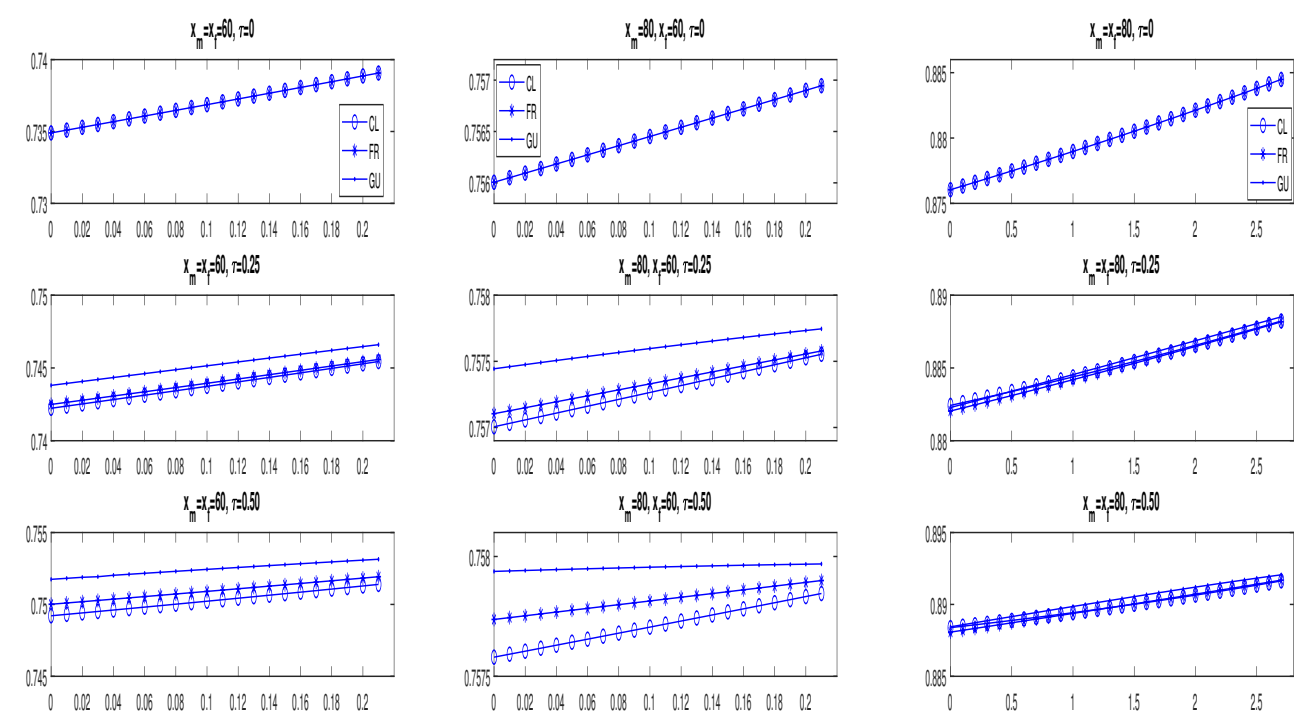

Figure 3: Last survival policy expected present value compared to $\lambda$ when the copula function changes. The dependence is measured by Kendall's $\tau$ coefficient. In the $\mathrm{x}$-axis there is the parameter $\lambda$ in percentage form. The parameters of marginal distributions are those estimated from our sample. The constant interest rate is $r=0.01$. Left: $x_{m}=x_{f}=60$; middle: $x_{m}=80, x_{f}=60$; right: $x_{m}=x_{f}=80$.

Such a policy does not distinguish between the case in which only one of the two individuals in the couple dies first and the case in which both spouses die simultaneously. We can introduce a policy which pays a different lump sum to the heirs in case of simultaneous death. Let $L$ denote the lump sum paid in case of simultaneous death and $l$ the one paid in case of the first single death. The expected 
present value of the future cash flow is

$$
\begin{aligned}
\bar{A}_{x_{m} x_{f}}^{S}(l, L) & =L \cdot \mathbb{E}\left[e^{-r \min \left(T^{m}, T^{f}\right)} \mathbf{1}_{\left\{T^{m}=T^{f}\right\}}\right]+l \cdot \mathbb{E}\left[e^{-r \min \left(T^{m}, T^{f}\right)} \mathbf{1}_{\left\{T^{m} \neq T^{f}\right\}}\right]= \\
& =L \cdot \int_{0}^{+\infty} e^{-r y} \bar{F}_{X^{m}, X^{f}}(y, y) f_{Z}(y) d y+l \cdot\left[\bar{A}_{x_{m} x_{f}}-\int_{0}^{+\infty} e^{-r y} \bar{F}_{X^{m}, X^{f}}(y, y) f_{Z}(y) d y\right]
\end{aligned}
$$

where we have used (11). Figure 4 shows its dynamics, again with respect to $\lambda$, where, as in the previous examples, the marginal distributions are those estimated by the sample. The entry ages are $x_{f}=x_{m}=60$ and the sum paid in case of the first single death, $l$, is fixed to 10 , whereas the sum paid in case of simultaneous death, $L$, can assume four values, 10, 10.5, 11, 12. Here, the copula is Frank and the levels of dependence are the same as before. We can observe that the behavior changes from decreasing to increasing as the value of the sum $L$ increases. More precisely, when $L$ becomes greater than 11 the curves turn to be increasing from decreasing and this property is accentuated with the increase in the level of dependence. This is clearly due to the fact that as the benefit in case of simultaneous death is much bigger than that in case of the first "single" death, then the impact on the premium of a greater intensity of simultaneous death becomes more relevant inducing an increase in the premium.

\section{Conclusions}

This paper introduces the Extended Marshall-Olkin model (see Pinto and Kolev, 2015) as a probabilistic model for the evaluation of insurance products written on the residual lifetimes of the two individuals in a couple. The approach is based on modeling the dependence between lifetimes using copula functions and combining the two approaches already considered in existing literature: i) the absolutely continuous copula based approach introduced in the seminal paper of Frees et al. (1996), where the copula is used to capture associations based on the environmental factors and lifestyle shared by the two lives; ii) the approach based on the classical Marshall-Olkin construction (see Denuit et al, 2006), in order to account for the occurrence of a fatal event causing the simultaneous death with a positive probability. The model is estimated on a Canadian insurer dataset and the impact on the premium calculation on policies written on the joint lives and on the event of simultaneous death are analyzed.

However, since the broken-heart effect is not related to the fatal exogenous catastrophic event, the modeling of this kind of association in the EMO model is the same as that in the classical absolutely continuous copula based approach and additional effort will be needed to further generalize the model in order to improve the modeling of this type of dependence. For example, one might consider an EMO model 

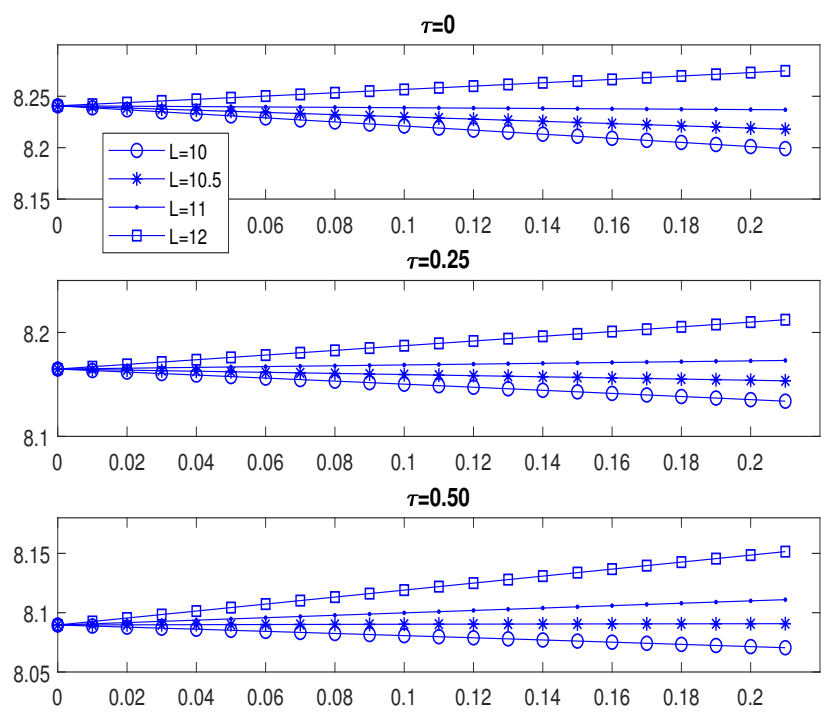

Figure 4: Last survival policy expected present value compared to $\lambda$ when the lump sum paid in case of simultaneous death changes. The copula function is Frank with three different levels of dependence measured by Kendall's $\tau$ coefficient. In the $\mathrm{x}$-axis there is the parameter $\lambda$ in percentage form. The parameters of marginal distributions are estimated from our sample. The constant interest rate is $r=0.01$. In each plot we set $l=10$ and $x_{f}=x_{m}=60$. 
generated by

$$
\left(T^{m}, T^{f}\right)=\left(\min \left(Y_{m} X^{m}, Z\right), \min \left(Y_{f} X^{f}, Z\right)\right),
$$

where $Y_{m}, Y_{f} \in(0,1)$ are random variables incorporating the broken-heart syndrome.

\section{Acknowledgments}

We are grateful to the helpful remarks and comments of both referees which highly improved the paper. The results have been obtained when the second author visited the Department of Statistics, University of Bologna during the first semester of 2018. The second author was supported by FAPESP grants 2017/14819-2 and 2013/07375-0.

\section{References}

[1] V. Asimit, E. Furman, R. Vernic (2016): "Statistical Inference for a New Class of Multivariate Pareto Distributions", Communications in Statistics - Simulation and Computation, 45(2), 456-471.

[2] Bowers N.L., Gerber U., Hickman J.C., Jones D.A., Nesbit C.J. (1997): Actuarial Mathematics. School of Actuaries.

[3] Carriere J. (2000): Bivariate Survival Models of Coupled Lives, Scandinavian Actuarial Journal, 17-31.

[4] Denuit M., Dhaene J., Le Bailly De Tilleghem C., Teghem S. (2001): Measuring the impact of dependence among insured lifelengths, Belgian Actuarial Bulletin, 1, 18-39.

[5] Denuit M., Frostig E., Levikson B. (2006): Shift in Interest Rate and Common Shock Model for Coupled Lives. Belgian Actuarial Bulletin 6, 1-4.

[6] Dufresne F. Hashrova E., Ratovomirija G., Toukourou Y. (2018): On age difference in joint lifetime modelling with life insurance annuity applications, Annals of Actuarial Sciences, 12, 2, 350-371.

[7] Frees E., Carriere J., Valdez E. (1996): Annuity Valuation with Dependent Mortality, Journal of Risk and Insurance, 63, 229-261.

[8] Gouriéroux C., Lu Y. (2015): Love and death: A Freund model with frailty, Insurance: Mathematics and Economics, 63, 191-203. 
[9] Ji M., Hardy M.R., Li J.S.-H. (2011): Markovian approaches to joint-life mortality, North American Actuarial Journal, 15, 3, 357-376.

[10] Karlis D. (2003): ML estimation for multivariate shock models via an EM algorithm, Ann. of the Institute of Statistical Mathematics, 155, 817-830.

[11] Kolev, N., Pinto, J. (2018): A weak version of bivariate lack of memory property. Brazilian Journal of Probability and Statistics, 32, 4, 873-906.

[12] Kundu D., Dey A. (2009): Estimating the parameters of the Marshall-Olkin bivariate Weibull distribution, Computational Statistics and Data Analysis, 57, 271-281.

[13] Kundu D., Gupta A. (2013): Bayes estimation for the Marshall-Olkin bivariate Weibull distribution by EM algorithm, Computational Statistics and Data Analysis, 53, 956-965.

[14] Lee H., Cha J.H. (2018): A dynamic bivariate common shock model with cumulative effect and its actuarial application, Scandinavian Actuarial Journal, DOI:10.1080/03461238.2018.1470562.

[15] Li X., Pellerey F. (2011): Generalized Marshall-Olkin distribution and related bivariate aging properties, Journal of Multivariate Analysis, 102(10), 13991409.

[16] Lu Y. (2017): Broken-Heart, common life, heterogeneity: analyzing the spousal mortality dependence, ASTIN Bulletin: the Journal of the IAA, 47(3), 837-874.

[17] Luciano E., Spreeuw J., Vigna E. (2008): Modelling Stochastic Mortality for Dependent Lives, Insurance: Mathematics and Economics, 43, 234-244.

[18] Luciano E., Spreeuw J., Vigna E. (2016): Spouses' Dependence across Generations and Pricing Impact on Reversionary Annuities, Risks, 4(2), 1-18.

[19] Marshall A.W., Olkin I. (1967): A Multivariate Exponential Distribution, J. Am. Stat. Assoc., 62, 30-44.

[20] Nelsen R.(2006): An Introduction to Copulas, Springer.

[21] Oakes D. (1989): Bivariate Survival Models Induced by Frailties, Journal of the American Statistical Association, 84, 406, 487-493.

[22] Pinto J., Kolev N. (2015): Extended Marshall-Olkin Model and Its Dual Version, In U. Cherubini, F. Durante, S. Mulinacci (eds.) Marshall-Olkin Distributions-Advances in Theory and Applications. Springer Proceedings in 
Mathematics and Statistics. Springer International Publishing Switzerland (2015), 87-113.

[23] Shemyakin A., Youn H. (2006): Copula Models of Joint Last Survivor Analysis, Applied Stochastic Models in Business and Industry, 22, 211-224.

[24] Shih J.H., Louis T.A. (1995): Inferences on the Association Parameter in Copula Models for Bivariate Survival Data, Biometrics, 51, 1384-1399.

[25] Sibuya M. (1960): Bivariate extreme statistics, Annals of the Institute of Statistical Mathematics, 11, 195-210.

[26] Spreeuw J. (2006): Types of Dependence and Time-dependent Association between two lifetimes in Single Parameter Copula Models, Scandinavian Actuarial Journal, 5, 286-309.

[27] Spreeuw, J., Wang X. (2008): Modelling the Short-term Dependence Between two Remaining Lifetimes, Cass Business School Discussion Paper.

[28] Spreeuw, J., Owadally I. (2013): Investigating the Broken-Heart Effect: a Model for Short-Term Dependence Between the Remaining Lifetimes of Joint Lives, Annals of Actuarial Science, 7(2), 236-257.

[29] Volinsky C.T., Raftery A.E.(2000): Bayesian information criterion for censored survival models, Biometrics, 56, 256-262.

[30] Youn H., Shemyakin A. (1999): Statistical aspects of joint life insurance pricing, Proceedings of the Business and Statistics Section of the American Statistical Association, 34-38.

[31] Youn H., Shemyakin A., Herman E. (2002): A re-examination of the joint mortality functions, North American Actuarial Journal, 6,1, 166-170. 